\title{
Convergence criteria and optimization techniques for beam moments
}

\author{
G Gbur and P S Carney \\ Department of Physics and Astronomy and the Rochester Theory Center for Optical Science
} and Engineering, University of Rochester, Rochester, NY 14627, USA

Received 13 January 1998, in final form 24 March 1998

\begin{abstract}
We consider the problem of the characterization of beams by moments of the field intensity in the aperture and its moments in the far field. The well known beam propagation factor, $M_{\mathrm{P}}^{2}$, is considered. We give convergence criteria for these factors and also discuss a new approach to optimization of the even moments of the far-field intensity.
\end{abstract}

\section{Introduction}

The beam propagation factor

$$
M_{\mathrm{P}}^{2}=4 \pi \sigma_{x} \sigma_{s}
$$

is frequently used as a simple measure of laser beam quality [1]. In this formula the variance of the intensity in the transverse plane at the beam waist and the variance of the far-field intensity distribution are denoted by $\sigma_{x}^{2}$ and $\sigma_{s}^{2}$, respectively. This factor has been the subject of much discussion since it was first introduced, mainly because of questions of computational stability [2] and because it diverges in some cases, i.e. the case of diffraction from a hard-edge aperture. Attempts have been made to overcome these problems by truncating the intensity distribution in the far field or otherwise limiting the range of integration when calculating the moments [3, 4]. Some authors $[3,5,6]$ have proposed additional standards involving higher-order moments. However, these higherorder moments diverge in certain cases just as the $M_{\mathrm{P}}^{2}$ factor does. Apart from convergence some authors have considered the problem of optimization of beam moments, subject to certain constraints $[10,11]$.

We address the question of convergence criteria for the beam propagation factor and for higher-order moments and discuss the problem of moment optimization. We will demonstrate that the convergence criteria play a crucial role in the solution of the optimization problem.

For simplicity, we will restrict our discussion to a beam with one transverse degree of freedom, which we denote by $x$; similar results hold for two transverse degrees of freedom. The field in the aperture plane will be specified by a function $f(x)$ which vanishes identically outside the range $a<x<b$. We assume that $f(x)$ is square integrable and has $N$ derivatives on this open interval. 


\section{Convergence of the second-order moment}

Apart from multiplicative factors, the field $F(s)$ in the far zone is related to the field $f(x)$ in the aperture by the expression

$$
F(s)=\int_{-\infty}^{\infty} f(x) \mathrm{e}^{-2 \pi \mathrm{i} s x} \mathrm{~d} x .
$$

The variance of the intensity in the far field is given by the formula

$$
\sigma_{s}^{2}=\left\langle s^{2}\right\rangle-\langle s\rangle^{2}
$$

where

$$
\left\langle s^{2}\right\rangle=\mathfrak{N} \int_{-\infty}^{\infty} s^{2}|F(s)|^{2} \mathrm{~d} s
$$

and $\mathfrak{N}$ is the normalization factor defined by the formula

$$
\mathfrak{N}^{-1}=\int_{-\infty}^{\infty}|F(s)|^{2} \mathrm{~d} s=\int_{-\infty}^{\infty}|f(x)|^{2} \mathrm{~d} x .
$$

We choose our coordinate system such that

$$
\langle s\rangle=0 \text {. }
$$

The second-order moment is then given by equation (2.3); it is this quantity for which we wish to determine convergence criteria. Since $f(x)$ is a bounded function of $x$ which vanishes outside the interval $a<x<b$ and the endpoints of the interval form a set of measure zero, the integral (2.1) is equivalent to

$$
F(s)=\lim _{\varepsilon \rightarrow 0} \int_{a+\varepsilon}^{b-\varepsilon} f(x) \mathrm{e}^{-2 \pi \mathrm{i} x x} \mathrm{~d} x .
$$

Integrating this expression repeatedly by parts we obtain an asymptotic series valid for large values of $|s|[8,9]$ :

$F(s) \sim \lim _{\varepsilon \rightarrow 0} \sum_{n=0}^{N-1}-(2 \pi \mathrm{i} s)^{-n-1}\left[\mathrm{e}^{-2 \pi \mathrm{i} s(b-\varepsilon)} f^{(n)}(b-\varepsilon)-\mathrm{e}^{-2 \pi \mathrm{i} s(a+\varepsilon)} f^{(n)}(a+\varepsilon)\right]+\mathrm{o}\left(s^{-N}\right)$

where $f^{(n)}(x)=\mathrm{d}^{n} f\left(x^{\prime}\right) /\left.\mathrm{d} x^{\prime n}\right|_{x^{\prime}=x}$ is the $n$th derivative of $f$. Since $F(s)$ is the Fourier transform of a function of finite support, it is bounded on any finite interval and thus the convergence of the second moment of $|F(s)|^{2}$ is determined solely by the behaviour for large $|s|$ of the integrand in equation (2.3). From equation (2.7), it then follows that

$$
\begin{aligned}
|F(s)|^{2} \sim \lim _{\varepsilon \rightarrow 0} & \sum_{m=0}^{N-1} \sum_{n=0}^{N-1}(2 \pi \mathrm{i} s)^{-n-m-2}(-1)^{m+1} \\
& \times\left[\mathrm{e}^{-2 \pi \mathrm{i} s(b-\varepsilon)} f^{(n)}(b-\varepsilon)-\mathrm{e}^{-2 \pi \mathrm{i} s(a+\varepsilon)} f^{(n)}(a+\varepsilon)\right] \\
& \times\left[\mathrm{e}^{2 \pi \mathrm{i} s(b-\varepsilon)} f^{(m) *}(b-\varepsilon)-\mathrm{e}^{2 \pi \mathrm{i} s(a+\varepsilon)} f^{(m) *}(a+\varepsilon)\right]+\mathrm{o}\left(s^{-N}\right) .
\end{aligned}
$$

The lowest-order term in negative powers of $s$ in the integrand $s^{2}|F(s)|^{2}$ is given by the term $m=n=0$ :

$\left[s^{2}|F(s)|^{2}\right]_{m=n=0}=\lim _{\varepsilon \rightarrow 0}(2 \pi)^{-2}\left|\mathrm{e}^{-2 \pi \mathrm{i} s(b-\varepsilon)} f(b-\varepsilon)-\mathrm{e}^{-2 \pi \mathrm{i} s(a+\varepsilon)} f(a+\varepsilon)\right|^{2}$. 
This term is oscillatory and non-negative for large $|s|$. Its contribution to the integral (2.3) will therefore only converge if

$$
\lim _{\varepsilon \rightarrow 0}[f(b-\varepsilon)]=0
$$

and

$$
\lim _{\varepsilon \rightarrow 0}[f(a+\varepsilon)]=0
$$

i.e. in order for the second-order moment of the far-zone intensity to be finite the field must approach zero continuously at the edges of the aperture. If equations (2.10) and (2.11) are satisfied, the next non-zero term in the expansion (2.8) is the $n=1, m=1$ term. This term and all higher-order terms in the integrand of equation (2.3) fall off as $s^{-2}$ or faster for large $|s|$ and therefore converge. Equations (2.10) and (2.11) constitute necessary and sufficient conditions for the variance of the far-zone intensity, and therefore the $M_{\mathrm{P}}^{2}$ factor, to exist.

\section{Convergence of higher even-order moments}

The preceding treatment may be extended to all moments of the form $\dagger$

$$
\left\langle s^{2 k}\right\rangle=\mathfrak{N} \int_{-\infty}^{\infty} s^{2 k}|F(s)|^{2} \mathrm{~d} s
$$

where $k$ is a positive integer. We may again make use of equation (2.8) and consider the $n=m=k-1$ term of the integrand of equation (3.1):

$$
\left[s^{2 k}|F(s)|^{2}\right]_{n=m=k-1}=\lim _{\varepsilon \rightarrow 0}(2 \pi)^{-2 k}\left|\mathrm{e}^{-2 \pi \mathrm{i} s(b-\varepsilon)} f^{(k-1)}(b-\varepsilon)-\mathrm{e}^{-2 \pi \mathrm{i}(a+\varepsilon)} f^{(k-1)}(a+\varepsilon)\right|^{2} .
$$

This term, like that given by equation (2.9), is oscillatory and is non-negative for large values of $|s|$ : it will add a diverging contribution to equation (3.1) unless

$$
\lim _{\varepsilon \rightarrow 0}\left[f^{(k-1)}(b-\varepsilon)\right]=0
$$

and

$$
\lim _{\varepsilon \rightarrow 0}\left[f^{(k-1)}(a+\varepsilon)\right]=0 .
$$

Consequently, for the $2 k$ th-order moment to exist, the $(k-1)$ st derivative of $f(x)$ must decrease continuously to zero at the edges of the aperture. Clearly, all lower order terms in $s$ must also vanish for the $2 k$ th-order moment to converge. This is only possible if

$$
\lim _{\varepsilon \rightarrow 0}\left[f^{(j)}(b-\varepsilon)\right]=0
$$

and

$$
\lim _{\varepsilon \rightarrow 0}\left[f^{(j)}(a+\varepsilon)\right]=0
$$

for all $j<k$. As in the case of the second moment, these conditions are also sufficient. Hence a necessary and sufficient condition for the $2 k$ th-order moment, $\left\langle s^{2 k}\right\rangle$, to exist is that the aperture field and its first $k-1$ derivatives go continuously to zero at the endpoints $x=a$ and $x=b$.

$\dagger$ The zeroth-order moment is defined without normalization so that it is proportional to the energy of the beam, $\left\langle s_{0}\right\rangle \equiv \int|F(s)|^{2} \mathrm{~d} s=\int|f(x)|^{2} \mathrm{~d} x$. 


\section{Convergence of the odd-order moments}

The odd-order moments are somewhat more difficult to deal with because a symmetric function $|F(s)|^{2}$ will always have vanishing odd-order moments, at least in the sense of the principal value [7]. Let us define a new function $A(s)$ by the expression

$$
A(s)=|F(s)|^{2}-|F(-s)|^{2} .
$$

Then

$$
\left\langle s^{2 k+1}\right\rangle=\mathfrak{N} \int_{0}^{\infty} A(s) s^{2 k+1} \mathrm{~d} s .
$$

It can be shown by arguments similar to those presented above that $A(s)$ has an asymptotic expansion

$$
A(s) \sim \sum_{j=0}^{N-1} \sum_{k \leqslant j} \frac{A_{j k}(s)}{(2 \pi \mathrm{i} s)^{j+k+2}}
$$

valid for large values of $|s|$. The functions $A_{j k}(s)$ are given by the expressions

$$
\begin{aligned}
& A_{k k}(s)=(-1)^{k} 4 \Im\left[f^{(k) *}(a+\varepsilon) f^{(k)}(b-\varepsilon)\right] \sin 2 \pi s(b-a-2 \varepsilon) \\
& A_{j k}(s)=(-1)^{k+1} 4 \Im\left[f^{(k)}(a+\varepsilon) f^{(j) *}(b-\varepsilon)+f^{(j)}(a+\varepsilon) f^{(k) *}(b-\varepsilon)\right] \\
& \times \sin 2 \pi s(b-a-2 \varepsilon) \text { for } j \neq k, \quad \text { even values of } j+k \\
&=(-1)^{k+1}\left\{4 \mathrm{i} \Im\left[f^{(k)}(b-\varepsilon) f^{(j) *}(b-\varepsilon)+f^{(j)}(a+\varepsilon) f^{(k) *}(a+\varepsilon)\right]\right. \\
&+4 \mathrm{i} \Im\left[f^{(k) *}(a+\varepsilon) f^{(j)}(b-\varepsilon)+f^{(j)}(a+\varepsilon) f^{(k) *}(b-\varepsilon)\right] \\
&\times \cos 2 \pi s(b-a-2 \varepsilon)\} \quad \text { for odd values of } j+k
\end{aligned}
$$

and $\mathfrak{s}$ denotes the imaginary part.

In order that a moment of order $2 m+1$ exists, the terms in the above expansion for which $j+k \leqslant 2 m$ must vanish order by order. This is a much less restrictive requirement than the conditions for the even moments. For instance, the condition

$$
\lim _{\varepsilon \rightarrow 0} f^{(j)}(a+\varepsilon)=\lim _{\varepsilon \rightarrow 0} f^{(j)}(b-\varepsilon)=0 \quad \text { for } \quad j \leqslant m
$$

is sufficient for the moments of order $2 m+1$ to exist, but it is not a necessary condition. Furthermore, if $f(x)$ is real or complex symmetric, all the odd moments will vanish identically.

Some remarks about convergence criteria seem appropriate here. It is not difficult to show, using the methods of the preceding sections, that discontinuities of the field or its derivatives anywhere within the aperture lead to divergent even-order moments. Specifically, a discontinuity in a derivative of order $M$ of the aperture function leads to a divergence of the $(2 M+2)$ th-order moment of the far-zone intensity. Evidently, care should be taken in using moments to characterize beams where convergence is not guaranteed a priori. For instance, in situations where only the field and its first derivative need be continuous, one would expect that only the second- and fourth-order moments will be useful in characterization.

\section{From convergence to optimization}

The problem of minimizing a ratio of moments, in our notation $\left\langle s^{2 n}\right\rangle /\left\langle s^{2 m}\right\rangle$, was discussed by McCutchen [10]. Using an approach based on higher-dimensional generalizations of the diffraction problem, McCutchen was able to obtain optimal solutions for cases where 
$n=m+1$. Asakura and Ueno [11] solved the problem of minimizing the second-order moment while holding the total energy (the zeroth-order moment) constant.

Using a calculus of variations approach, we will consider here the general problem of minimizing $\left\langle s^{2 n}\right\rangle$, while holding $N$ other even-order moments constant. These $N$ moments may be of lesser or higher order than the quantity to be minimized.

We note that the $2 k$ th moment may be expressed in the form $\dagger$

$$
\left\langle s^{2 k}\right\rangle=\left(\frac{1}{2 \pi}\right)^{2 k} \mathfrak{N} \int\left|f^{(k)}(x)\right|^{2} \mathrm{~d} x
$$

where $\mathfrak{N}$ is defined in equation (2.4). Using this representation for the moments, we begin with a functional of the form

$$
\mathcal{J}=\int\left\{\mathfrak{N}\left|f^{(n)}(x)\right|^{2}+\sum_{k=1}^{N} \lambda_{k} \mathfrak{N}\left|f^{\left(m_{k}\right)}(x)\right|^{2}\right\} \mathrm{d} x
$$

with isoperimetric constraint equations [12]

$$
\mathfrak{N}\left(\frac{1}{2 \pi}\right)^{2 m_{k}} \int\left|f^{\left(m_{k}\right)}(x)\right|^{2} \mathrm{~d} x=\mu_{k} .
$$

Here $2 m_{k}$ and $\mu_{k}$ are the order and the value respectively of the $k$ th constrained moment. We may rewrite the functional (5.2) as

$$
\mathcal{J}=\int\left\{\sum_{k=1}^{N+1} \lambda_{k}\left[\int\left|f\left(x^{\prime}\right)\right|^{2} \mathrm{~d} x^{\prime}\right]^{-1}\left|f^{\left(m_{k}\right)}(x)\right|^{2}\right\} \mathrm{d} x
$$

with

$$
\lambda_{N+1} \equiv 1 \quad m_{N+1} \equiv n .
$$

We will assume $f$ to be real; the final differential equations which we obtain are also valid when $f$ is complex. Taking the first variation of the functional $\mathcal{J}$ defined in equation (5.4) we find that

$$
\delta \mathcal{J}=2 \int\left\{\sum_{k=1}^{N+1} \lambda_{k} \mathfrak{N}\left[-\left\langle s^{2 m_{k}}\right\rangle f(x)(\delta f)+f^{\left(m_{k}\right)}(x)(\delta f)^{\left(m_{k}\right)}\right]\right\} \mathrm{d} x .
$$

One might try to integrate expression (5.6) by parts, set $\delta \mathcal{J}$ equal to zero and thus obtain a differential equation for $f$. Because of the explicit appearance of $\left\langle s^{\left(2 m_{k}\right)}\right\rangle$ in equation (5.6), this is only possible if the convergence criteria are satisfied for all even-order moments up to the highest-order even moment involved. More explicitly, if we set

$$
M=\max \left\{m_{k}\right\}
$$

then the aperture field and first $M-1$ derivatives of the field must go continuously to zero at the edges of the aperture. Our optimization problem therefore requires that an additional $2 M$ constraints be satisfied, in the form of $2 M$ boundary conditions on the aperture field and its derivatives. If these conditions are satisfied, we obtain the Euler-Lagrange equation:

$$
\sum_{k=0}^{N+1}(-1)^{m_{k}} \lambda_{k} f^{\left(2 m_{k}\right)}(x)=0
$$

where, for convenience, we have defined

$$
\lambda_{0} \equiv \sum_{k=1}^{N+1} \lambda_{k}\left\langle s^{2 m_{k}}\right\rangle \quad m_{0} \equiv 0 .
$$

$\dagger$ See the first footnote. 
Equation (5.8) has solutions which depend upon $2 M+N+2$ parameters, $2 M$ constants of integration and $N+2$ undetermined multipliers, $\lambda_{k}$. Equations (5.3) and (5.5) provide $N+1$ constraints, while equation (5.9) provides another constraint. This is generally not enough to specify all the free parameters. It is now necessary to invoke the $2 M$ boundary conditions given by equations (3.5) and (3.6), which must be imposed to satisfy the convergence criteria. These conditions form a set of $2 M$ linear, homogeneous equations.

It is clear from the preceding discussion that the convergence criteria are important for solving the optimization problem. It should be noted, though, that because our constraints are isoperimetric, the $2 M+N+2$ equations for the $2 M+N+2$ unknowns do not form a linear system and thus the equations may not have a solution in the space of $N+1$ Lagrange multipliers. In the case that no moment is specified, $N=0$, and a solution always exists. However, one cannot solve a problem where moments are constrained to values less than some minimum possible value. For instance, consider the problem of minimizing the fourth-order moment $\left\langle s^{4}\right\rangle$. If one specifies the energy $\left\langle s^{0}\right\rangle$, then one cannot also require that the second-order moment $\left\langle s^{2}\right\rangle$ be less than the minimum value obtained by solving the variational problem to minimize the second moment with the energy fixed.

One possible solution is to weaken the constraints. We could seek to minimize the expression $\left\langle s^{4}\right\rangle /\left\langle s^{2}\right\rangle$, or perhaps $\left\langle s^{4}\right\rangle\left\langle s^{2}\right\rangle$ without further constraints. Such minimization problems, involving minimization of products of the form

$$
\mathcal{J}^{\prime}=\prod_{k=1}^{N^{\prime}}\left\langle s^{2 m_{k}}\right\rangle^{\nu_{k}}
$$

(of which the McCutchen problem [10] is a special case), can be solved using methods equivalent to those specified in the above discussion. The Euler-Lagrange equation for such a functional results, after a long calculation, in a differential equation which is identical to the differential equation (5.8) found for the functional $\mathcal{J}$, but with $N^{\prime}=N+1$, and with the Lagrange multipliers given in terms of the as yet undetermined moments:

$$
\lambda_{k}=(2 \pi)^{-2 m_{k}} v_{k}\left\langle s^{2 m_{k}}\right\rangle^{-1}
$$

and

$$
\lambda_{0}=-\sum_{k=1}^{N^{\prime}} v_{k} .
$$

The same boundary conditions must be applied and if this still leaves undetermined constants then we can seek the extrema of $\mathcal{J}^{\prime}$ in the space of the undetermined multipliers $\{\lambda\}$ by means of the normal calculus of several variables,

$$
\nabla_{\lambda} \mathcal{J}^{\prime}=0
$$

The possible optimization problems of this type are numerous and the appropriate choice of the functional $\mathcal{J}^{\prime}$ depends upon the intended application.

\section{Some examples}

We will now illustrate the methods developed above by some examples. In all of the following cases we will assume that $a=-\delta$ and $b=\delta$. 


\subsection{The second-order moment}

We consider minimization of the second-order moment, while holding the energy $\left\langle s^{0}\right\rangle=\mu$ constant. We must then solve the second-order differential equation which results from minimizing

$$
\mathcal{J}=\left\langle s^{2}\right\rangle+\lambda\left\langle s^{0}\right\rangle
$$

with the appropriate boundary conditions, i.e.

$$
\lim _{\varepsilon \rightarrow 0} f( \pm \delta \mp \varepsilon)=0
$$

and using the energy constraint.

The methods outlined above result in an optimum solution given by the expression

$$
f(x)=\sqrt{\mu / \delta} \cos \left(\frac{\pi x}{2 \delta}\right)
$$

which agrees with the solution found by Asakura and Ueno [11]. The resulting far field is given by the expression

$$
F(s)=\frac{4 \sqrt{\mu \delta} \cos 2 \pi \delta s}{\pi(1-4 \delta s)(1+4 \delta s)} .
$$

Using this result we find the minimum value of the second-order moment for a fixed energy is

$$
\left\langle s^{2}\right\rangle_{\min }=\frac{1}{16 \delta^{2}} .
$$

The value of the propagation factor for this example is $M_{\mathrm{P}}^{2}=1.136 \dagger$.

\subsection{The ratio $\left\langle s^{4}\right\rangle /\left\langle s^{2}\right\rangle$}

The functional to be considered here is

$$
\mathcal{J}^{\prime}=\left\langle s^{4}\right\rangle\left\langle s^{2}\right\rangle^{-1} \text {. }
$$

With the same interval as above and requiring that the solution satisfies the convergence criteria, one can find that the aperture function which minimizes this quantity is given by the expression

$$
f(x)=\Gamma \cos ^{2}\left(\frac{\pi x}{2 \delta}\right)
$$

where $\Gamma$ is an undetermined constant. The far field is then given by the expression

$$
F(s)=\frac{\Gamma \sin 2 \pi \delta s}{2 \pi s(1-2 \delta s)(1+2 \delta s)}
$$

and the functional $\mathcal{J}^{\prime}$ defined by equation (6.6) takes the value

$$
\mathcal{J}_{\min }^{\prime}=\frac{1}{4 \delta^{2}}
$$

and the propagation factor is $M_{\mathrm{P}}^{2}=1.026$.

It should be noted that this function satisfies our convergence criteria for the fourthorder moment, while the solution obtained in the previous section only satisfies the criteria for the second-order moment and, in fact, diverges for the fourth-order moment.

$\dagger$ For comparison, in the idealized case of a Gaussian beam propagating from an infinite aperture, the propagation factor takes on its minimum value of unity. 


\subsection{The fourth-order moment}

The problem of minimizing the fourth-order moment $\left\langle s^{4}\right\rangle$ differs from those encountered in the previous two examples in that it is not solvable by either the Asakura and Ueno method [11], or by the method outlined by McCutchen [10]. The functional appropriate to this situation is given by the expression

$$
\mathcal{J}^{\prime}[f]=\frac{\int\left|f^{\prime \prime}(x)\right|^{2} \mathrm{~d} x}{(2 \pi)^{4} \int|f(x)|^{2} \mathrm{~d} x} .
$$

We find from equation (5.8) that in this case the aperture function must satisfy the differential equation

$$
f^{(4)}(x)=\lambda f(x)
$$

with the boundary conditions

$$
\lim _{\varepsilon \rightarrow 0} f( \pm \delta \mp \varepsilon)=\lim _{\varepsilon \rightarrow 0} f^{\prime}( \pm \delta \mp \varepsilon)=0 .
$$

In solving this system of equations we find solutions of two basic forms. Let us denote by $\lambda_{s n}$ solutions of the transcendental equation

$$
\tan \left(\lambda_{s n} \delta\right)=-\tanh \left(\lambda_{s n} \delta\right)
$$

The functions

$$
\phi_{n}(x)=\Gamma\left[\cos \left(\lambda_{s n} \delta\right) \cosh \left(\lambda_{s n} x\right)-\cosh \left(\lambda_{s n} \delta\right) \cos \left(\lambda_{s n} x\right)\right]
$$

are then solutions of the differential equation and satisfy the boundary conditions (6.12). Let us denote by $\lambda_{\text {an }}$ solutions of the transcendental equation

$$
\tan \left(\lambda_{a n} \delta\right)=\tanh \left(\lambda_{a n} \delta\right)
$$

The functions

$$
\psi_{n}=\Gamma\left[\sinh \left(\lambda_{a n} \delta\right) \sin \left(\lambda_{a n} x\right)-\sin \left(\lambda_{a n} \delta\right) \sinh \left(\lambda_{a n} x\right)\right]
$$

are then also solutions of the differential equation and satisfy the boundary conditions.

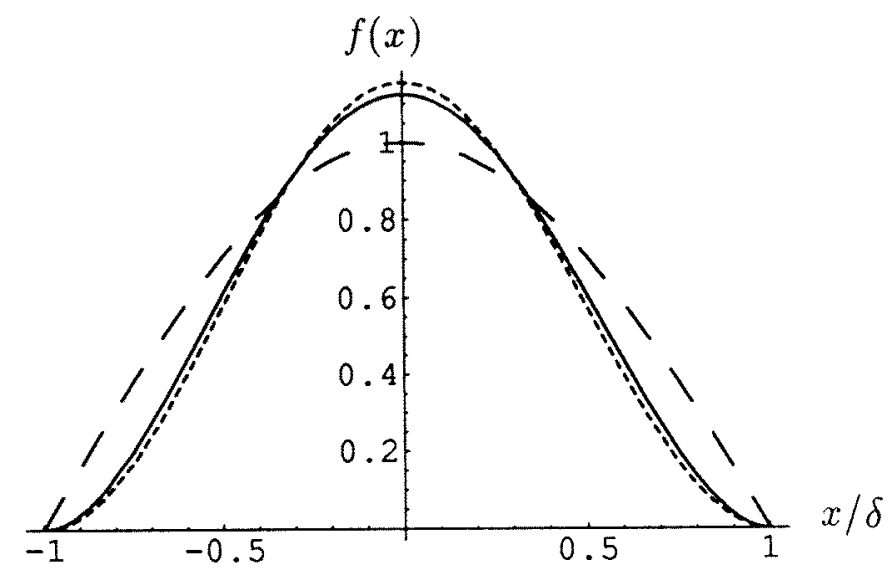

Figure 1. The aperture functions minimizing $\left\langle s^{2}\right\rangle,\left\langle s^{4}\right\rangle /\left\langle s^{2}\right\rangle$ and $\left\langle s^{4}\right\rangle$ shown as long-broken, short-broken and full curves, respectively. 


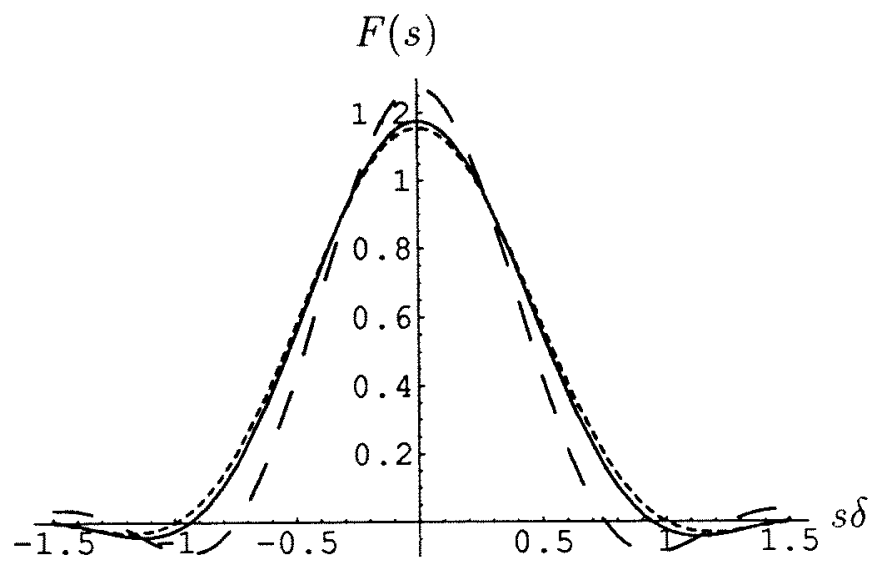

Figure 2. The angular spectra resulting from the aperture functions.

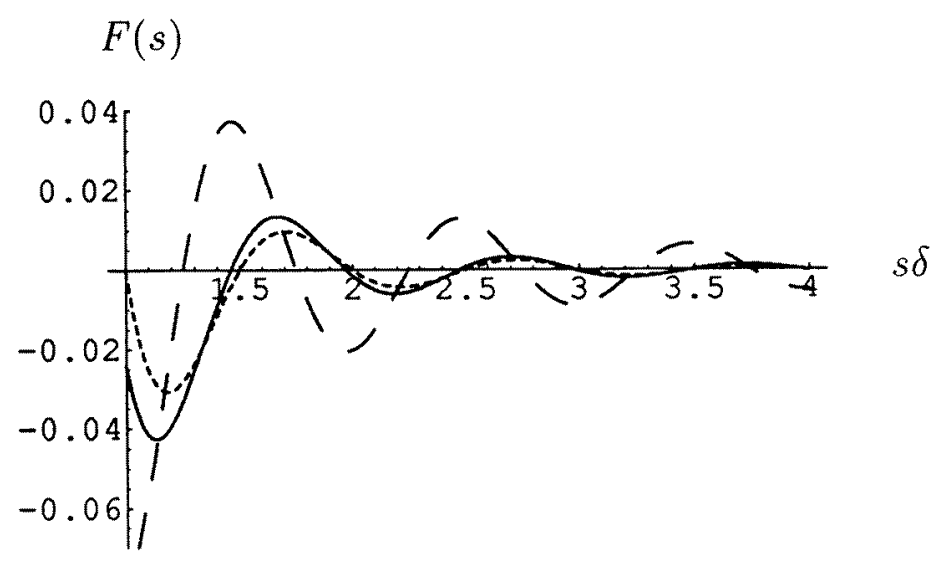

Figure 3. The tails of the angular spectra resulting from the aperture functions.

We find that, in this case,

$$
\mathcal{J}^{\prime}\left[\phi_{n}\right]=\left(\frac{\lambda_{s n}}{2 \pi}\right)^{4}
$$

and that

$$
\mathcal{J}^{\prime}\left[\psi_{n}\right]=\left(\frac{\lambda_{a n}}{2 \pi}\right)^{4}
$$

It can be seen that $\lambda_{s 1}$ is the lowest eigenvalue and thus the true global (nontrivial) minimum corresponds to the $\phi_{1}$ solution which has a propagation factor of $M_{\mathrm{P}}^{2}=1.037$.

The three aperture functions found in this section are shown in figure 1 and the corresponding far fields are shown in figures 2 and 3. The functions have been normalized so that in all three cases the energy is $\left\langle s^{0}\right\rangle=\delta$. 


\section{Acknowledgments}

The authors would like to thank Dr Emil Wolf for his useful suggestions and comments, and also wish to congratulate him on reaching this early milestone in his career. We also wish to thank Dr Pierre-André Bélanger and Dr Claude Paré for their helpful comments on this work. This research was supported by the Air Force Office of Scientific Research under grants F49620-96-10400 and F49620-97-1-0482 and by the Department of Energy under grant DE-FG02-90E12.

\section{References}

[1] Siegman A E 1990 New developments in laser resonators Proc. SPIE ed D A Holmes (Chicago, IL: SPIE) pp 2-14

[2] Lawrence G N 1994 Proposed international standard for laser-beam quality falls short Laser Focus World July $109-14$

[3] Bélanger P, Champagne Y and Paré C 1994 Beam propagation factor of diffracted laser beams Opt. Commun. $105233-42$

[4] Martinez-Herrero R and Mejias P M 1993 Second-order spatial characterization of hard-edge diffracted beams Opt. Lett. 18 1669-71

[5] Martinez-Herrero R, Majias P M, Sanchez M and Neira J L H 1992 Third- and fourth-order parametric characterization of partially coherent beams propagating through ABCD optical systems Opt. Quantum Electron. 24 S1021-6

[6] Weber H 1992 Propagation of higher-order intensity moments in quadratic-index media Opt. Quantum Electron. 24 S1027-49

[7] Bremermann H 1965 Distributions, Complex Variables and Fourier Transforms (Reading, MA: AddisonWesley)

[8] Bleistein N and Handelsman R A 1975 Asymptotic Expansion of Integrals (New York: Holt, Rinehart and Winston)

[9] Erdelyi A 1956 Asymptotic Expansions (New York: Dover)

[10] McCutchen C W 1969 Two families of apodization problems J. Opt. Soc. Am. 59 1163-71

[11] Asakura T and Ueno T 1976 Apodization for minimizing the second moment of the intensity distribution in the Fraunhofer diffraction pattern Nouv. Rev. Opt. 7 199-203

[12] Lanczos C 1986 The Variational Principles of Mechanics 4th edn (New York: Dover) 